\title{
A numerical approach with residual error estimation for solution of high-order linear differential-difference equations by using Gegenbauer polynomials
}

\author{
${ }^{1 *}$ Tuğçe Mollaoğlu , ${ }^{2}$ Mehmet Sezer \\ ${ }^{1}$ Department of Mathematics, Faculty of Science, Manisa Celal Bayar University \\ Manisa, Turkey, tugce.mollaoglu@hotmail.com \\ ${ }^{2}$ Department of Mathematics, Faculty of Science, Manisa Celal Bayar University \\ Manisa, Turkey, mehmet.sezer@cbu.edu.tr \\ ${ }^{*}$ Corresponding author \\ Received: 17th November 2016 \\ Accepted: 27th December 2016 \\ DOI: http://dx.doi.org/10.18466/cbujos.302638
}

\begin{abstract}
The main aim of this study is to apply the Gegenbauer polynomials for the solution of high-order linear differential difference equations with functional arguments under initial-boundary conditions. The technique we have used is essentially based on the truncated Gegenbauer series and its matrix representations along with collocation points. Also, by using the Mean-Value Theorem and residual function, an efficient error estimation technique is proposed and some illustrative examples are presented to demonstrate the validity and applicability of the method.
\end{abstract}

Keywords - Gegenbauer polynomials; Differential-difference equations; Collocation method;

Matrix method; Error estimation; Residual function.

\section{Introduction}

Differential-difference equations [1-10], which are a class of functional differential equations, have been treated as models of some physical phenomenon. When a mathematical model is developed for a physical system, it is usually assumed that all of the variables, such as space and time, are continuous. This assumption leads to a realistic and justified approximation of the real variables of the system. However, for some of the physical systems, these continuous variable assumptions can not be made. Then differential-difference equations have played an important role in modelling problems that appear in various branches of science; e.g. mechanical engineering, condensed matter, biophysics, mathematical statistics and control theory. In recent years, the studies of differentialdifference equations are developed very rapidly and intensively. It is well known that linear differential-difference equations have been considered by many authors, and have been used in the applications of difference models to problems in biology, physics and engineering.

In this study, the basic ideas of the mentioned studies are developed to obtain the approximate solutions of high-order linear differentialdefference equation with functional arguments and variable coefficients in the form

$$
\sum_{k=0}^{m} P_{k}(x) y^{(k)}(x)+\sum_{j=0}^{\mathrm{J}} Q_{j}(x) y^{(j)}(\alpha x+\beta)=f(x),
$$


CBÜ Fen Bil. Dergi., Cilt 13, Say1 1 2017, 39-49 s

$$
\mathrm{J} \leq m
$$

under the mixed conditions

$$
\begin{gathered}
\sum_{k=0}^{m-1}\left(a_{k l} y^{(k)}(a)+b_{k l} y^{(k)}(b)\right)=\lambda_{l}, \\
l=0,1, \ldots, m-1
\end{gathered}
$$

Here $P_{k}(x), Q_{j}(x)$ and $f(x)$ are known functions defined on the interval $a \leq x \leq b$;

$\alpha, \beta, a_{l k}, b_{l k}$ and $\lambda_{l}$ are appropriate constants; $y(x)$ is an unknown function to be determined. The aim of this study is to get the solution of the problem (1) - (2) as the truncated Gegenbauer series defined by

$$
\begin{gathered}
y(x) \cong y_{N}(x)=\sum_{n=0}^{N} a_{n} \mathrm{G}_{n}(x, \lambda), \\
N \geq m,-1 \leq x \leq 1
\end{gathered}
$$

where $a_{n}, n=0,1, \ldots, \mathrm{N}$ are unknown coefficients; $\mathrm{G}_{n}(x, \lambda)$, classical polynomials orthogonal on the interval $(-1,1)$ with respect to the weight function $w \rightarrow\left(1-x^{2}\right)^{\lambda-\frac{1}{2}}$

$\left(\lambda>-\frac{1}{2}\right)$, denote the Gegenbauer polynomials defined by [16-20]

$$
\mathrm{G}_{n}(x, \lambda)=\sum_{k=0}^{\left[\frac{n}{2}\right]}(-1)^{k} \frac{(\lambda)_{n-k}}{k !(n-2 k) !}(2 x)^{n-2 k}
$$

or recursively

$$
\begin{aligned}
n \mathrm{G}_{n}(x, \lambda)= & 2 x(\lambda+n-1) \mathrm{G}_{n-1}(x, \lambda) \\
& -(n+2 \lambda-2) \mathrm{G}_{n-2}(x, \lambda), n \geq 2
\end{aligned}
$$

with $\mathrm{G}_{0}(x, \lambda)=1$ and $\mathrm{G}_{1}(x, \lambda)=2 \lambda x$. On the other hand, by using (4), the first four Gegenbauer polynomials are given by

$$
\mathrm{G}_{0}(x, \lambda)=1
$$$$
\mathrm{G}_{1}(x, \lambda)=(-1)^{0} \frac{2^{1}(\lambda)_{1}}{0 ! 1 !} x^{1}
$$

$\mathrm{G}_{2}(x, \lambda)=(-1)^{1} \frac{2^{0}(\lambda)_{1}}{1 ! 0 !} x^{0}+(-1)^{0} \frac{2^{2}(\lambda)_{2}}{0 ! 2 !} x^{2}$
CBU J. of Sci., Volume 13, Issue 1, 2017 p 39-49

$$
\mathrm{G}_{3}(x, \lambda)=(-1)^{1} \frac{2^{1}(\lambda)_{2}}{1 ! 1 !} x^{1}+(-1)^{0} \frac{2^{3}(\lambda)_{3}}{0 ! 3 !} x^{3}
$$

\section{Fundamental matrix relations}

Let us consider the solution $\mathrm{y}(x)$ of Eq. (1) defined by the truncated Gegenbauer series (3):

$y(x) \cong y_{N}(x)=\sum_{n=0}^{N} a_{n} \mathbf{G}_{n}(x, \lambda), N \geq m,-1 \leq x \leq 1$.

Then we can convert the finite series (3) to the matrix form as, for $n=0,1, \ldots, N$

$$
\begin{gathered}
y(x) \cong y_{N}(x)=\mathbf{G}(x, \lambda) \mathbf{A} \\
\mathbf{G}(x, \lambda)=\left[\begin{array}{llll}
G_{0}(x) & G_{1}(x) & \ldots & G_{N}(x)
\end{array}\right] \\
\mathbf{A}=\left[\begin{array}{llll}
a_{0} & a_{1} & \ldots & a_{N}
\end{array}\right]^{T} .
\end{gathered}
$$

On the other hand, by using the relation (4), the matrix $\mathbf{G}(x, \lambda)$ is obtained as

$$
\mathbf{G}(x, \lambda)=\mathbf{X}(x) \mathbf{T}(\lambda)
$$

where

$$
\mathbf{X}(x)=\left[\begin{array}{lllll}
1 & x & x^{2} & \ldots & x^{N}
\end{array}\right] .
$$

If $\mathrm{N}$ is odd,

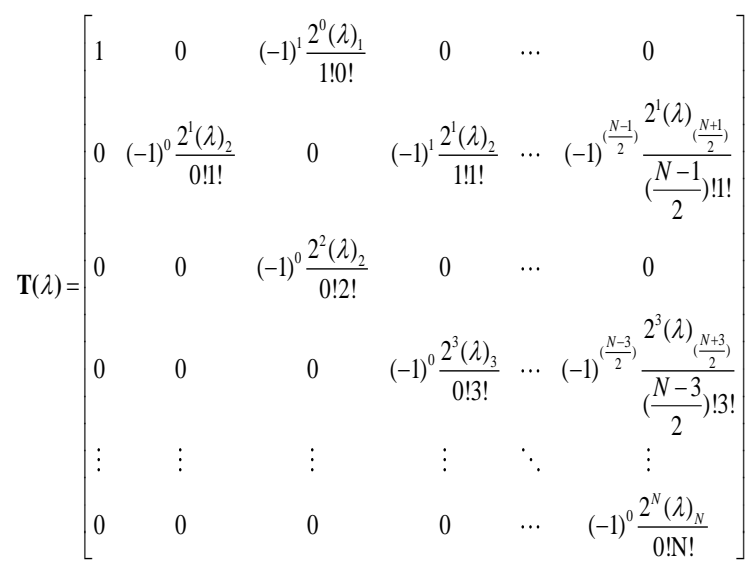

If $\mathrm{N}$ is even, 
CBÜ Fen Bil. Dergi., Cilt 13, Sayı 1 2017, 39-49 s

$$
\mathbf{T}(\lambda)=\left[\begin{array}{cccccc}
1 & 0 & (-1)^{1} \frac{2^{0}(\lambda)_{1}}{1 ! 0 !} & 0 & \cdots & (-1)^{\left(\frac{N}{2}\right)} \frac{2^{0}(\lambda)^{\left(\frac{N}{2}\right)}}{\left(\frac{N}{2}\right) ! 1 !} \\
0 & (-1)^{0} \frac{2^{1}(\lambda)_{2}}{0 ! 1 !} & 0 & (-1)^{1} \frac{2^{1}(\lambda)_{2}}{1 ! ! !} & \cdots & 0 \\
0 & 0 & (-1)^{0} \frac{2^{2}(\lambda)_{2}}{0 ! 2 !} & 0 & \cdots & (-1)^{\left(\frac{N-2}{2}\right)} \frac{2^{2}(\lambda)^{\left(\frac{N+2}{2}\right)}}{\left(\frac{N-2}{2}\right) ! 2 !} \\
0 & 0 & 0 & (-1)^{0} \frac{2^{3}(\lambda)_{3}}{0 ! 3 !} & \cdots & 0 \\
\vdots & \vdots & \vdots & \vdots & \ddots & \vdots \\
0 & 0 & 0 & 0 & \cdots & (-1)^{0} \frac{2^{N}(\lambda)_{N}}{0 ! N !}
\end{array}\right]
$$

Also, it is clearly seen from (6) that the relation between the matrix $\mathbf{G}(x, \lambda)$ and its derivative $\mathbf{G}^{\prime}(x, \lambda)$ is

$$
\mathbf{G}^{\prime}(x, \lambda)=\mathbf{X}^{\prime}(x) \mathbf{T}(\lambda)=\mathbf{X}(x) \mathbf{B} \mathbf{T}(\lambda)
$$

where

$$
\mathbf{X}^{\prime}(x)=\mathbf{X}(x) \mathbf{B}
$$

and that repeating the process

$$
\mathbf{G}^{(k)}(x, \lambda)=\mathbf{X}(x) \mathbf{B}^{k} \mathbf{T}(\lambda), k=0,1,2, \ldots, m
$$

where

$$
\mathbf{B}=\left[\begin{array}{ccccc}
0 & 1 & 0 & \cdots & 0 \\
0 & 0 & 2 & \cdots & 0 \\
\vdots & \vdots & \vdots & \cdots & \vdots \\
0 & 0 & 0 & \cdots & N \\
0 & 0 & 0 & \cdots & 0
\end{array}\right], \mathbf{B}^{0}=\left[\begin{array}{ccccc}
1 & 0 & 0 & \cdots & 0 \\
0 & 1 & 0 & \cdots & 0 \\
0 & 0 & 1 & \cdots & 0 \\
\vdots & \vdots & \vdots & \ddots & \vdots \\
0 & 0 & 0 & \cdots & 1
\end{array}\right]
$$

From the matrix relations (5),(6) and (7), it follows that

$$
y(x) \cong y_{N}(x)=\mathbf{G}(x, \lambda) \mathbf{A}=\mathbf{X}(x) \mathbf{T}(\lambda) \mathbf{A}
$$

and

$$
\begin{aligned}
& y^{(k)}(x) \cong y_{N}{ }^{(k)}(x)=\mathbf{G}^{(k)}(x, \lambda)=\mathbf{X}^{(k)}(x) \mathbf{T}(\lambda) \mathbf{A} \\
&=\mathbf{X}(x) \mathbf{B}^{k} \mathbf{T}(\lambda) \mathbf{A}, \mathrm{k}=0,1, \ldots
\end{aligned}
$$

By substituting $x \rightarrow \alpha x+\beta, k \rightarrow j$ into the relation (8), we get $j=0,1, \ldots$

$$
\begin{aligned}
y^{(j)}(\alpha x+\beta) & =\mathbf{X}(\alpha x+\beta) \mathbf{B}^{j} \mathbf{T}(\lambda) \\
& =\mathbf{X}(x) \mathbf{B}(\alpha, \beta) \mathbf{B}^{j} \mathbf{T}(\lambda) \mathbf{A}
\end{aligned}
$$

so that, for $\alpha, \beta \neq 0$,
CBU J. of Sci., Volume 13, Issue 1, 2017 p 39-49

$$
\mathbf{B}(\alpha, \beta)=\left[\begin{array}{ccccc}
\left(\begin{array}{l}
0 \\
0
\end{array}\right) \alpha^{0} \beta^{0} & \left(\begin{array}{l}
1 \\
0
\end{array}\right) \alpha^{0} \beta^{1} & \left(\begin{array}{l}
2 \\
0
\end{array}\right) \alpha^{0} \beta^{2} & \ldots & \left(\begin{array}{c}
N \\
0
\end{array}\right) \alpha^{0} \beta^{N} \\
0 & \left(\begin{array}{l}
1 \\
1
\end{array}\right) \alpha^{1} \beta^{0} & \left(\begin{array}{l}
2 \\
1
\end{array}\right) \alpha^{1} \beta^{1} & \ldots & \left(\begin{array}{c}
N \\
1
\end{array}\right) \alpha^{1} \beta^{N-1} \\
0 & 0 & \left(\begin{array}{c}
2 \\
2
\end{array}\right) \alpha^{2} \beta^{0} & \ldots & \left(\begin{array}{c}
N \\
2
\end{array}\right) \alpha^{2} \beta^{N-2} \\
\vdots & \vdots & \vdots & \ddots & \vdots \\
0 & 0 & 0 & \cdots & \left(\begin{array}{l}
N \\
N
\end{array}\right) \alpha^{N} \beta^{0}
\end{array}\right]
$$

for $\alpha \neq 0, \beta=0$,

$$
\mathbf{B}(\alpha, 0)=\left[\begin{array}{ccccc}
0 \\
\left(\begin{array}{l}
0
\end{array}\right) \alpha^{0} & 0 & 0 & \ldots & 0 \\
0 & \left(\begin{array}{l}
1 \\
1
\end{array}\right) \alpha^{1} & 0 & \ldots & 0 \\
0 & 0 & \left(\begin{array}{l}
2 \\
2
\end{array}\right) \alpha^{2} & \cdots & 0 \\
\vdots & \vdots & \vdots & \ddots & \vdots \\
0 & 0 & 0 & \cdots & \left(\begin{array}{l}
N \\
N
\end{array}\right) \alpha^{N}
\end{array}\right]
$$

\section{Gegenbauer collocation method}

For constructing the fundamental matrix equation, we first consider the collocation points defined by, for $i=0,1, \ldots, N$,

$$
x_{i}=a+\frac{b-a}{N} i,(\text { Standard })
$$

or

$$
\begin{gathered}
x_{i}=\frac{b+a}{2}-\frac{b-a}{2} \cos \left(\frac{\pi i}{N}\right),(\text { Chebyshev } \\
- \text { Lobatto })
\end{gathered}
$$

Then, by using the collocation points (10) into (1), we have the system of the equations

$$
\sum_{k=0}^{m} P_{k}\left(x_{i}\right) y^{(k)}\left(x_{i}\right)+\sum_{j=0}^{\mathrm{J}} Q_{j}\left(x_{i}\right) y^{(j)}\left(\alpha x_{i}+\beta\right)=f\left(x_{i}\right)
$$

or briefly the corresponding matrix equation

$$
\sum_{k=0}^{m} \mathbf{P}_{k} \mathbf{Y}^{(k)}+\sum_{j=0}^{\mathrm{J}} \boldsymbol{Q}_{j} \mathbf{Y}^{(j)}(\alpha, \beta)=\mathbf{F}
$$

where

$$
\begin{aligned}
& \mathbf{P}_{k}=\operatorname{diag}\left[P_{k}\left(x_{0}\right) \quad P_{k}\left(x_{1}\right) \quad \cdots \quad P_{k}\left(x_{N}\right)\right] \\
& \mathbf{Q}_{j}=\operatorname{diag}\left[Q_{j}\left(x_{0}\right) \quad Q_{j}\left(x_{1}\right) \quad \cdots \quad Q_{j}\left(x_{N}\right)\right]
\end{aligned}
$$


$\mathbf{Y}^{(k)}=\left[\begin{array}{c}y^{(k)}\left(x_{0}\right) \\ y^{(k)}\left(x_{1}\right) \\ \vdots \\ y^{(k)}\left(x_{N}\right)\end{array}\right], \mathbf{Y}^{(j)}(\alpha, \beta)=\left[\begin{array}{c}y^{(j)}\left(\alpha x_{0}+\beta\right) \\ y^{(j)}\left(\alpha x_{1}+\beta\right) \\ \vdots \\ y^{(j)}\left(\alpha x_{N}+\beta\right)\end{array}\right]$,

$\mathbf{F}=\left[\begin{array}{c}f\left(x_{0}\right) \\ f\left(x_{1}\right) \\ \vdots \\ f\left(x_{N}\right)\end{array}\right]$.

On the other hand, by substituting the collacation points (10) into (8) and (9), we obtain the matrix relations

$\mathbf{Y}^{(k)}=\left[\begin{array}{c}y^{(k)}\left(x_{0}\right) \\ y^{(k)}\left(x_{1}\right) \\ \vdots \\ y^{(k)}\left(x_{N}\right)\end{array}\right]=\left[\begin{array}{c}\mathbf{X}\left(x_{0}\right) \mathbf{B}^{k} \mathbf{T}(\lambda) \mathbf{A} \\ \mathbf{X}\left(x_{1}\right) \mathbf{B}^{k} \mathbf{T}(\lambda) \mathbf{A} \\ \vdots \\ \mathbf{X}\left(x_{N}\right) \mathbf{B}^{k} \mathbf{T}(\lambda) \mathbf{A}\end{array}\right]=\mathbf{X B}^{k} \mathbf{T}(\lambda) \mathbf{A}$

and

$$
\begin{aligned}
\mathbf{Y}^{(j)}(\alpha, \beta) & =\left[\begin{array}{c}
y^{(j)}\left(\alpha x_{0}+\beta\right) \\
y^{(j)}\left(\alpha x_{1}+\beta\right) \\
\vdots \\
y^{(j)}\left(\alpha x_{N}+\beta\right)
\end{array}\right] \\
& =\left[\begin{array}{c}
\mathbf{X}\left(x_{0}\right) \mathbf{B}(\alpha, \beta) \mathbf{B}^{j} \mathbf{T}(\lambda) \mathbf{A} \\
\mathbf{X}\left(x_{1}\right) \mathbf{B}(\alpha, \beta) \mathbf{B}^{j} \mathbf{T}(\lambda) \mathbf{A} \\
\vdots \\
\mathbf{X}\left(x_{N}\right) \mathbf{B}(\alpha, \beta) \mathbf{B}^{j} \mathbf{T}(\lambda) \mathbf{A}
\end{array}\right]=\mathbf{X B}(\alpha, \beta) \mathbf{B}^{j} \mathbf{T}(\lambda) \mathbf{A}
\end{aligned}
$$

so that

$$
\mathbf{X}=\left[\begin{array}{c}
\mathbf{X}\left(x_{0}\right) \\
\mathbf{X}\left(x_{1}\right) \\
\vdots \\
\mathbf{X}\left(x_{N}\right)
\end{array}\right]=\left[\begin{array}{cccc}
1 & x_{0} & \cdots & x_{0}{ }^{N} \\
1 & x_{1} & \cdots & x_{1}{ }^{N} \\
\vdots & \vdots & \ddots & \vdots \\
1 & x_{N} & \cdots & x_{N}{ }^{N}
\end{array}\right]
$$

Therefore, the fundamental matrix equation (11) becomes

$$
\left\{\sum_{k=0}^{m} \mathbf{P}_{k} \mathbf{X B}^{k}+\sum_{j=0}^{\mathrm{J}} \boldsymbol{Q}_{j} \mathbf{X B}(\alpha, \beta) \mathbf{B}^{j}\right\} \mathbf{T}(\lambda) \mathbf{A}=\mathbf{F}
$$

Now we can find the fundamental matrix form for the conditions (2), by using the relation (8), as follows:

$$
\begin{gathered}
\sum_{k=0}^{m-1}\left(a_{k l} \mathbf{X}(\mathrm{a})+b_{k l} \mathbf{X}(b)\right) \mathbf{B}^{k} \mathbf{T}(\lambda) \mathbf{A}=\lambda_{l}, \\
l=0,1, \ldots, m-1
\end{gathered}
$$

Then, we can write the following fundamental matrix equations (12) and (13) corresponding to Eq.(1) and the conditions (2), respectively,
$\mathbf{W A}=\mathbf{F}$ or $[\mathbf{W} ; \mathbf{F}]$

and

$\mathbf{U}_{l} \mathbf{A}=\lambda_{l}$ or $\left[\mathbf{U}_{l} ; \lambda_{l}\right], l=0,1, \ldots, m-1$

where

$$
\begin{gathered}
\mathbf{W}=\left\{\sum_{k=0}^{m} \mathbf{P}_{k} \mathbf{X B}^{k}+\sum_{j=0}^{\mathrm{J}} \boldsymbol{Q}_{j} \mathbf{X B}(\alpha, \beta) \mathbf{B}^{j}\right\} \mathbf{T}(\lambda) \\
\mathbf{U}_{l}=\sum_{k=0}^{m-1}\left(a_{k l} \mathbf{X}(\mathrm{a})+b_{k l} \mathbf{X}(b)\right) \mathbf{B}^{k} \mathbf{T}(\lambda),
\end{gathered}
$$

Consequently, to obtain the solution of Eq.(1) under the conditions (2), by replacing the row matrix (15) by the last (or any) $\mathrm{m}$ rows of the augmented matrix (14), we have the required matrix

$$
[\widetilde{\mathbf{W}} ; \tilde{\mathbf{F}}] \text { or } \widetilde{\mathbf{W}} \mathbf{A}=\tilde{\mathbf{F}} .
$$

If $\operatorname{rank} \widetilde{\mathbf{W}}=\operatorname{rank}[\widetilde{\mathbf{W}} ; \tilde{\mathbf{F}}]=\mathrm{N}+1$, then we can write $\mathbf{A}=(\widetilde{\mathbf{W}})^{-1} \widetilde{\mathbf{F}}$. Thus the matrix A (thereby the coefficients $\left.a_{0}, a_{1}, \ldots, a_{N}\right)$ is uniquely determined. Eq.(1) under the conditions (2) has a unique solution. Hence, the problem (1) - (2) has a unique solution in terms of Gegenbauer polinomials in the form (3).

\section{Accuracy of solutions and residual error estimation}

We can easily check the accuracy of the obtained solutions as follows. Since the truncated Gegenbauer series (3) is approximate solution of (1), when the function $y_{N}(x)$ and its derivatives are substituted in Eq.(1), the resulting equation must be satisfied approximately ;

that is, for $x=x_{q} \in[a, b], q=0,1, \ldots$

$$
\begin{aligned}
R_{N}\left(x_{q}\right) & =\sum_{k=0}^{m} P_{k}\left(x_{q}\right) y^{(k)}\left(x_{q}\right) \\
& +\sum_{j=0}^{\mathrm{J}} Q_{j}\left(x_{q}\right) y^{(j)}\left(\alpha x_{q}+\beta\right)-f\left(x_{q}\right) \cong 0, \mathrm{~J} \leq m
\end{aligned}
$$

or 
CBÜ Fen Bil. Dergi., Cilt 13, Say1 1 2017, 39-49 s

$R_{N}\left(x_{q}\right) \leq 10^{-k_{q}}$, $\left(k_{q}\right.$ is any positive integer $)$.

If $\max 10^{-k_{q}}=10^{-k}$ is prescribed, then the truncation limit $\mathrm{N}$ is increased until the difference $R_{N}\left(x_{q}\right)$ at each of the points becomes smaller than the prescribed $10^{-k}$. Therefore, if $R_{N}\left(x_{q}\right) \rightarrow 0$ when $\mathrm{N}$ is sufficiently large enough then the error decreases.

On the other hand, by means of the residual function defined by $R_{N}(x)$ and the mean value of the function $\left|R_{N}(x)\right|$ on the interval [a, b], the accuracy of the solution can be controlled and the error can be estimated $[7-9,14-21]$. Thus, we can estimate the upper bound of the mean error $\overline{R_{N}}$ as follows :

$$
\left|\int_{a}^{b} R_{N}(x) d x\right| \leq \int_{a}^{b}\left|R_{N}(x)\right| d x
$$

and

$$
\begin{gathered}
\int_{a}^{b}\left|R_{N}(x)\right| d x=(b-a)\left|R_{N}(c)\right|, a \leq c \leq b \\
\Rightarrow\left|\int_{a}^{b} R_{N}(x) d x\right|=(b-a)\left|R_{N}(c)\right| \\
\Rightarrow(b-a)\left|R_{N}(c)\right| \leq \int_{a}^{b}\left|R_{N}(x)\right| d x \\
\Downarrow \\
\left|R_{N}(c)\right| \leq \frac{\int_{a}^{b}\left|R_{N}(x)\right| d x}{b-a}=\overline{R_{N}}
\end{gathered}
$$

\section{Numerical Examples}

In this section, several numerical examples are given to illustrate the accuracy and effectiveness properties of the method, and all of them are performed on the computer using a program written in Matlab. The absolute errors in the tables are computed by the relation
CBU J. of Sci., Volume 13, Issue 1, 2017 p 39-49

$$
\left|e_{N}(x)\right|=\left|y(x)-y_{N}(x)\right|
$$

at selected points.

Example 1. Let us consider differentialdifference equation

$y^{\prime}(x)=x y(x)+\frac{1}{2} e^{-x} y(x-1)+f(x),-1 \leq x \leq 1$

with the initial condition $y(0)=1$. Here,

$P_{0}=-x, P_{1}=1, Q_{0}=-\frac{1}{2} e^{-x}, \alpha=1, \beta=-1$,

$\lambda=1, f(x)=e^{x}-x e^{x}-\frac{1}{2} e^{-1}$. The exact solution of problem is $y(x)=e^{x}$.

Firstly, let us compute the approximate solution in the form defined by

$$
y_{2}(x)=\sum_{n=0}^{2} a_{n} G_{n}(x, 1)
$$

The set of the collocation points for $\mathrm{N}=2$ is calculated as

$$
\left\{x_{0}=-1, x_{1}=0, x_{2}=1\right\}
$$

and from Eq. (12), the fundamental matrix equation of the problem is written as

$$
\left\{\sum_{k=0}^{1} P_{k} \mathbf{X B}^{k}+\sum_{j=0}^{0} Q_{j} \mathbf{X B}(1,-1) \mathbf{B}^{j}\right\} \mathbf{T}(1) \mathbf{A}=\mathbf{F}
$$

After some operations, we obtain following matrices :

$$
\begin{aligned}
& \mathbf{P}_{0}=\left[\begin{array}{rrr}
-1 & 0 & 0 \\
0 & 0 & 0 \\
0 & 0 & 1
\end{array}\right], \mathbf{P}_{1}=\left[\begin{array}{lll}
1 & 0 & 0 \\
0 & 0 & 0 \\
0 & 0 & 1
\end{array}\right], \mathbf{X}=\left[\begin{array}{rrr}
1 & -1 & 1 \\
1 & 0 & 0 \\
1 & 1 & 1
\end{array}\right], \\
& \mathbf{Q}_{0}=\left[\begin{array}{ccc}
-632 / 465 & 0 & 0 \\
0 & -1 / 2 & 0 \\
0 & 0 & -268 / 1457
\end{array}\right] \text {, } \\
& \mathbf{T}=\left[\begin{array}{ccc}
1 & 0 & -1 \\
0 & 2 & 0 \\
0 & 0 & 4
\end{array}\right], \mathbf{B}(1,-1)=\left[\begin{array}{rrr}
1 & -1 & 1 \\
0 & 1 & -2 \\
0 & 0 & 1
\end{array}\right] \\
& \mathbf{B}=\left[\begin{array}{lll}
0 & 1 & 0 \\
0 & 0 & 2 \\
0 & 0 & 0
\end{array}\right], \mathbf{B}^{\mathbf{0}}=\left[\begin{array}{lll}
1 & 0 & 0 \\
0 & 1 & 0 \\
0 & 0 & 1
\end{array}\right], \mathbf{F}=\left[\begin{array}{c}
1001 / 1814 \\
1189 / 1457 \\
-268 / 1457
\end{array}\right]
\end{aligned}
$$

The augmented matrix for the fundamental matrix equation can be written as 


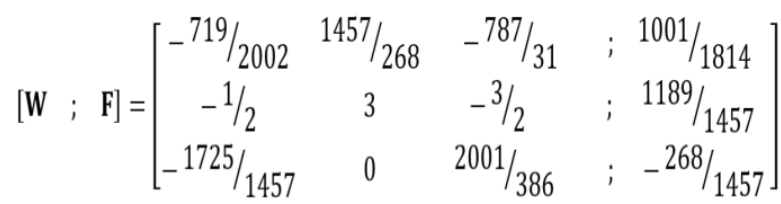

From Eq.(15), we obtain the matrix form for the initial condition:

$$
\left[\mathbf{U}_{0} \quad ; \quad \lambda_{0}\right]=\left[\begin{array}{lllll}
1 & 0 & -1 & ; & 1
\end{array}\right]
$$

From Eq. (16), the desired augmented matrix based on the condition is calculated as

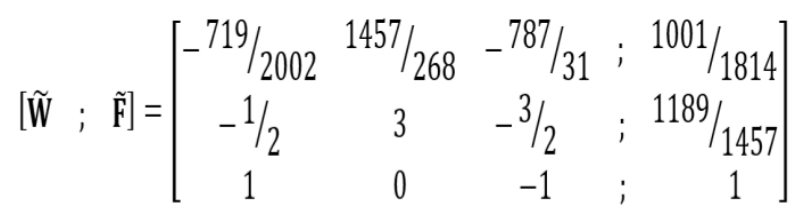

Solving this system, the unknown coefficient matrix is obtained as

$$
\mathbf{A}=\left[\begin{array}{lll}
1953 / 1831 & 1530 / 3167 & 367 / 5508
\end{array}\right]^{\mathrm{T}}
$$

Therefore, we obtain the approximate solution $y_{2}=1+0.9662 x+0.2665 x^{2}$.

For $N=2,3,4,5$ the obtained approximate solutions and the upper bounds of the mean error $\overline{R_{N}}$ are

$y_{3}=1+1.0262 x+0.5017 x^{2}+0.1049 x^{3}$,

$y_{4}=1+1.0004 x+0.5093 x^{2}+0.1682 x^{3}+0.0281 x^{4}$

$y_{5}=1+0.9996 x+0.5000 x^{2}+0.1690 x^{3}$

$+0.0420 x^{4}+0.0058 x^{5}$

$\overline{R_{2}}=0.32926, \overline{R_{3}}=5.1293 \times 10^{-2}$,

$\overline{R_{4}}=8.2124 \times 10^{-3}, \overline{R_{5}}=1.8751 \times 10^{-3}$

Taking $N=2,3,4,5$, the obtained approximate solutions are compared with the exact solutions in Fig.1. The absolute errors are demonstrated in Table 1.

The residual error functions of Example 1 are shown in Fig. 2.

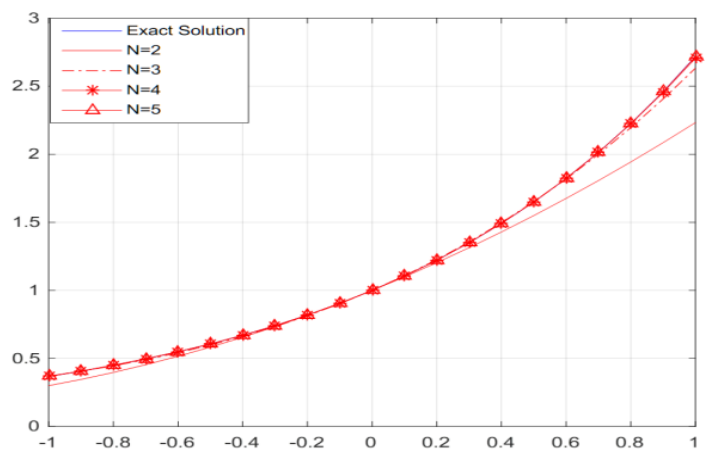

Fig.1. Numerical and exact solutions of Example 1 for $\mathrm{N}=2,3,4,5$,

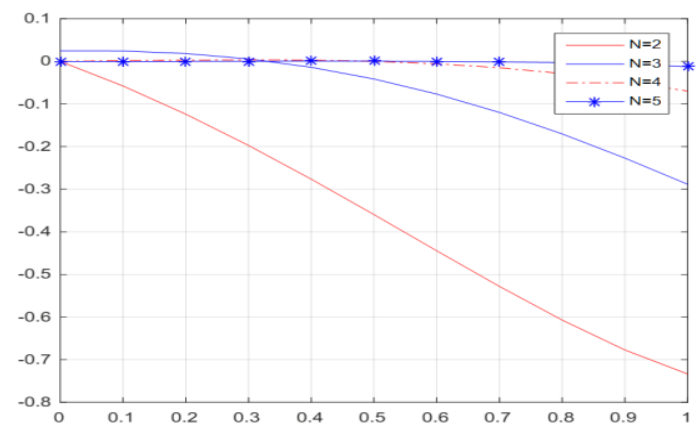

Fig.2. Residual error functions of Example 1 for $\mathrm{N}=2,3,4,5$.

Table 1. Comparison of the absolute errors of Example 1 for $\mathrm{N}=2,3,4,5$.

\begin{tabular}{cccccc}
\hline $\mathrm{x}_{\mathrm{i}}$ & $y\left(x_{\mathrm{i}}\right)=e^{\mathrm{x}_{i}}$ & $\mid e_{2}\left(\mathrm{x}_{\mathrm{i}}\right)$ & $\left|e_{3}\left(\mathrm{x}_{\mathrm{i}}\right)\right|$ & $e_{4}\left(\mathrm{x}_{\mathrm{i}}\right) \mid$ & $e_{3}\left(\mathrm{x}_{\mathrm{i}}\right) \mid$ \\
\hline-1 & 0.3679 & 0.0676 & 0.0027 & $9.2056 \mathrm{e}-04$ & $2.7944 \mathrm{e}-04$ \\
-0.5 & 0.6065 & 0.0230 & 0.0073 & 0.0013 & $1.1910 \mathrm{e}-05$ \\
0 & 1 & 0 & 0 & 0 & 0 \\
0.5 & 1.6487 & 0.0990 & 0.0029 & 0.0016 & $9.9783 \mathrm{e}-06$ \\
1 & 2.7183 & 0.4856 & 0.0855 & 0.0123 & 0.0019 \\
\hline
\end{tabular}

Example 2. Let us consider the differential equation with proportional delays

$$
\begin{aligned}
& y^{\prime \prime \prime}(x)-x y^{\prime \prime}(2 x)+y^{\prime}+y\left(\frac{x}{2}\right)=x \cos (2 x)+\cos \left(\frac{x}{2}\right) \\
& \text { with the initial conditions } y(0)=1,-1 \leq x \leq 1
\end{aligned}
$$

$y^{\prime}(0)=0, y^{\prime \prime}(0)=-1$. Here, $P_{1}=1, P_{3}=1$,

$Q_{0}=1, \alpha_{0}=\frac{1}{2}, \beta_{0}=0, Q_{2}=-x, \alpha_{1}=2$,

$\beta_{1}=0, \lambda=1, f(x)=x \cos (2 x)+\cos \left(\frac{x}{2}\right)$.

The exact solution of problem is $y(x)=\cos (x)$. 
CBÜ Fen Bil. Dergi., Cilt 13, Sayı 1 2017, 39-49 s

Firstly, the approximate solution $y_{3}(x)$ by the truncated Gegenbauer series for $N=3$ is given by

$$
y_{3}(x)=\sum_{n=0}^{3} a_{n} G_{n}(x, 1)
$$

Then, the set of the collocation points for $N=3$ is calculated as

$$
\left\{x_{0}=-1, x_{1}=-\frac{1}{3}, x_{2}=\frac{1}{3}, x_{3}=1\right\}
$$

and from Eq. (12), the fundamental matrix equation of the problem is written as

$$
\left\{\sum_{k=0}^{3} P_{k} \mathbf{X B}^{k}+\sum_{j=0}^{1} Q_{j} \mathbf{X B}(\alpha, \beta) \mathbf{B}^{j}\right\} \mathbf{T}(\lambda) \mathbf{A}=\mathbf{F}
$$

After some operations, we obtain following matrices:

$$
\begin{aligned}
& \mathbf{P}_{1}=\left[\begin{array}{llll}
1 & 0 & 0 & 0 \\
0 & 1 & 0 & 0 \\
0 & 0 & 1 & 0 \\
0 & 0 & 0 & 1
\end{array}\right], \mathbf{P}_{3}=\left[\begin{array}{llll}
1 & 0 & 0 & 0 \\
0 & 1 & 0 & 0 \\
0 & 0 & 1 & 0 \\
0 & 0 & 0 & 1
\end{array}\right] \\
& \mathbf{Q}_{0}=\left[\begin{array}{llll}
1 & 0 & 0 & 0 \\
0 & 1 & 0 & 0 \\
0 & 0 & 1 & 0 \\
0 & 0 & 0 & 1
\end{array}\right], \mathbf{T}(1)=\left[\begin{array}{cccc}
1 & 0 & -1 & 0 \\
0 & 2 & 0 & -4 \\
0 & 0 & 4 & 0 \\
0 & 0 & 0 & 8
\end{array}\right], \\
& \mathbf{Q}_{2}=\left[\begin{array}{cccr}
1 & 0 & 0 & 0 \\
0 & 1 / 3 & 0 & 0 \\
0 & 0 & -1 / 3 & 0 \\
0 & 0 & 0 & -1
\end{array}\right], \mathbf{B}(2,0)=\left[\begin{array}{llll}
1 & 0 & 0 & 0 \\
0 & 2 & 0 & 0 \\
0 & 0 & 4 & 0 \\
0 & 0 & 0 & 8
\end{array}\right], \\
& \mathbf{B}(1 / 2,0)=\left[\begin{array}{cccr}
1 & 0 & 0 & 0 \\
0 & 1 / 2 & 0 & 0 \\
0 & 0 & 1 / 4 & 0 \\
0 & 0 & 0 & 1 / 8
\end{array}\right], \mathbf{B}=\left[\begin{array}{llll}
0 & 1 & 0 & 0 \\
0 & 0 & 2 & 0 \\
0 & 0 & 0 & 3 \\
0 & 0 & 0 & 0
\end{array}\right] \text {, } \\
& \mathbf{F}=\left[\begin{array}{l}
392 / 303 \\
575 / 794 \\
659 / 528 \\
347 / 752
\end{array}\right], \mathbf{X}=\left[\begin{array}{cccc}
1 & -1 & 1 & -1 \\
1 & -1 / 3 & 1 / 9 & -1 / 27 \\
1 & 1 / 3 & 1 / 9 & 1 / 27 \\
1 & 1 & 1 & 1
\end{array}\right]
\end{aligned}
$$

The augmented matrix for the fundamental matrix equation is calculated as

$$
[\mathbf{W} ; \quad \mathbf{F}]=\left[\begin{array}{cccccc}
1 & 1 & 0 & -27 & ; & 392 / 303 \\
1 & 5 / 3 & -8 / 9 & 989 / 27 & ; & 575 / 794 \\
1 & 7 / 3 & -8 / 9 & 955 / 27 & ; & 659 / 528 \\
1 & 3 & 0 & -29 & ; & 347 / 752
\end{array}\right]
$$

CBU J. of Sci., Volume 13, Issue 1, 2017 p 39-49

From Eq.(15), we obtain the matrix form for the initial conditions:

$$
\begin{aligned}
& {\left[\begin{array}{lll}
\mathbf{U}_{0} & ; & \lambda_{0}
\end{array}\right]=\left[\begin{array}{llllll}
1 & 0 & -1 & 0 & ; & 1
\end{array}\right]} \\
& {\left[\begin{array}{lll}
\mathbf{U}_{1} ; & \lambda_{1}
\end{array}\right]=\left[\begin{array}{llllll}
0 & 2 & 0 & -4 & ; & 0
\end{array}\right]} \\
& {\left[\begin{array}{lll}
\mathbf{U}_{2} & ; & \lambda_{2}
\end{array}\right]=\left[\begin{array}{llllll}
0 & 0 & 8 & 0 & ; & -1
\end{array}\right]}
\end{aligned}
$$

From Eq. (16), the desired augmented matrix based on the condition is calculated as

$$
[\widetilde{\mathbf{W}} \quad ; \quad \tilde{\mathbf{F}}]=\left[\begin{array}{rrrrrc}
1 & 1 & 0 & -27 & ; & 392 / 303 \\
1 & 0 & -1 & 0 & ; & 1 \\
0 & 2 & 0 & -4 & ; & 0 \\
0 & 0 & 8 & 0 & ; & -1
\end{array}\right]
$$

Solving this system, the unknown coefficient matrix is obtained as

$$
\mathbf{A}=\left[\begin{array}{lllll}
7 / 8 & -203 / 6060 & -1 / 8 & -203 / 12120
\end{array}\right]^{\mathrm{T}}
$$

Therefore, we obtain the approximate solution $y_{3}=1-0.5000 x^{2}-0.1340 x^{3}$

For $N=4,5$ by using the same procedure, the following solutions are obtained :

$$
\begin{aligned}
y_{4} & =1-0.5000 x^{2}-0.0494 x^{3}+0.0132 x^{4}, \\
y_{5} & =1-0.5000 x^{2}+0.0029 x^{3}+0.0477 x^{4} \\
& +0.0056 x^{5} .
\end{aligned}
$$

Taking $N=3,4,5$ the obtained approximate solutions are compared with the exact solutions in Fig. 3; The absolute errors are demonstrated in Table 2. The graphics of the residual error functions are shown in Fig. 4 . 
CBÜ Fen Bil. Dergi., Cilt 13, Sayı 1 2017, 39-49 s

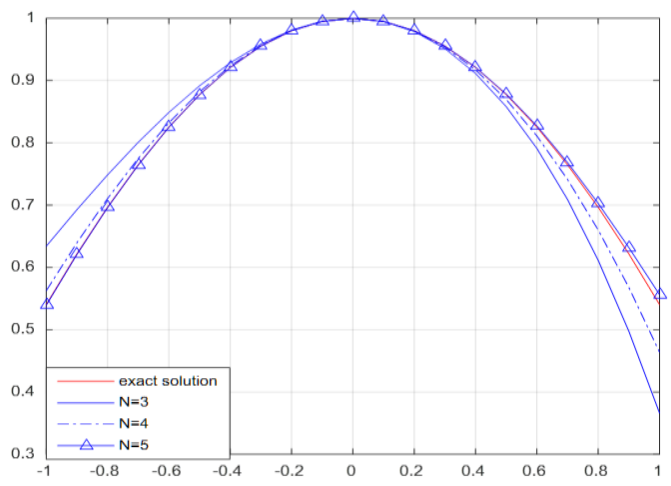

Fig. 3. Numerical and exact solutions of Example 2 for $\mathrm{N}=3,4,5$.

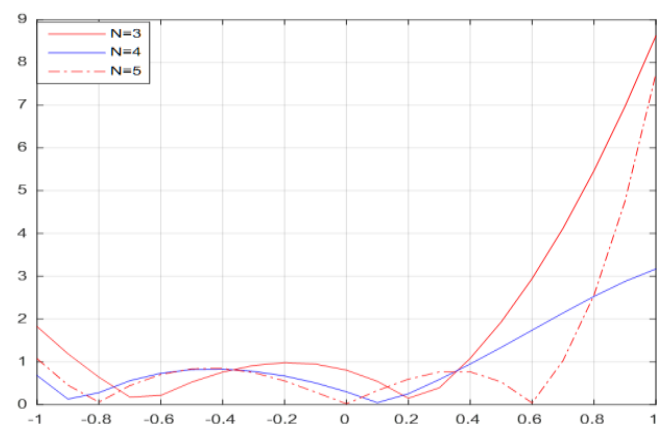

Fig.4. Residual error functions of Example 2 for $\mathrm{N}=3,4,5$

Table 2. Comparison of the absolute errors of Example 2 for $\mathrm{N}=3,4,5$.

\begin{tabular}{ccccc}
\hline $\mathrm{x}_{\mathrm{i}}$ & $y\left(x_{i}\right)=\cos \left(x_{i}\right)$ & $\left|e_{3}\left(\mathrm{x}_{i}\right)\right|$ & $\left|e_{4}\left(\mathrm{x}_{i}\right)\right|$ & $\left|e_{5}\left(\mathrm{x}_{i}\right)\right|$ \\
\hline-1 & 0.5403 & 0.0937 & $2.2 \mathrm{e}-02$ & $1.1 \mathrm{e}-03$ \\
-0.5 & 0.8776 & 0.0142 & $4.4 \mathrm{e}-3$ & $1.3 \mathrm{e}-04$ \\
0 & 1 & 0 & 0 & 0 \\
0.5 & 0.8776 & 0.0193 & $7.9 \mathrm{e}-3$ & $9.3 \mathrm{e}-04$ \\
1 & 0.5403 & 0.1743 & $7.6 \mathrm{e}-2$ & $1.59 \mathrm{e}-2$ \\
\hline
\end{tabular}

Example 3. Let us consider differential-

difference equation

$$
y^{\prime}(x)+2 y(x)-y(x-1)=f(x),-1 \leq x \leq 1
$$

with the initial condition $y(0)=1$. Here, $P_{0}=$ 2, $P_{1}=1, Q_{0}=-1, \alpha=1, \beta=-1, \lambda=1, f(x)=$ $3 e^{x}-e^{-x-1}$. The exact solution of problem is $y(x)=e^{x}$.

Firstly, let us compute the approximate solution $y_{2}(x)$ by the truncated Gegenbauer series for $N=2$ is given by

$$
y_{2}(x)=\sum_{n=0}^{2} a_{n} G_{n}(x, 1)
$$

CBU J. of Sci., Volume 13, Issue 1, 2017 p 39-49

The set of the collocation points for $N=2$ is calculated as

$$
\left\{x_{0}=-1, x_{1}=0, x_{2}=1\right\}
$$

and from Eq.(12), the fundamental matrix equation of the problem is written as

$$
\left\{\sum_{k=0}^{1} P_{k} \mathbf{X B}^{k}+\sum_{j=0}^{0} Q_{j} \mathbf{X B}(1,-1) \mathbf{B}^{j}\right\} \mathbf{T}(1) \mathbf{A}=\mathbf{F}
$$

After some operations, we obtain following matrices:

$$
\begin{aligned}
& \mathbf{P}_{0}=\left[\begin{array}{lll}
2 & 0 & 0 \\
0 & 2 & 0 \\
0 & 0 & 2
\end{array}\right], \mathbf{P}_{1}=\left[\begin{array}{lll}
1 & 0 & 0 \\
0 & 1 & 0 \\
0 & 0 & 1
\end{array}\right], \mathbf{X}=\left[\begin{array}{rrr}
1 & -1 & 1 \\
1 & 0 & 0 \\
1 & 1 & 1
\end{array}\right], \\
& \mathbf{T}=\left[\begin{array}{rrr}
1 & 0 & -1 \\
0 & 2 & 0 \\
0 & 0 & 4
\end{array}\right], \mathbf{B}=\left[\begin{array}{lll}
0 & 1 & 0 \\
0 & 0 & 2 \\
0 & 0 & 0
\end{array}\right], \mathbf{B}^{\mathbf{0}}=\left[\begin{array}{lll}
1 & 0 & 0 \\
0 & 1 & 0 \\
0 & 0 & 1
\end{array}\right], \\
& \mathbf{Q}_{0}=\left[\begin{array}{rrr}
-1 & 0 & 0 \\
0 & -1 & 0 \\
0 & 0 & -1
\end{array}\right], \mathbf{F}=\left[\begin{array}{c}
94 / 907 \\
3835 / 1457 \\
1644 / 205
\end{array}\right],
\end{aligned}
$$$$
\mathbf{B}(1,-1)=\left[\begin{array}{rrr}
1 & -1 & 1 \\
0 & 1 & -2 \\
0 & 0 & 1
\end{array}\right] .
$$

The augmented matrix for the fundamental matrix equation can be written as

$$
[\mathbf{W} \quad ; \quad \mathbf{F}]=\left[\begin{array}{ccccc}
1 & 2 & -17 & ; & 94 / 907 \\
1 & 4 & -5 & ; & 3835 / 1457 \\
1 & 6 & 15 & ; & 1644 / 205
\end{array}\right]
$$

From Eq.(15), we obtain the matrix form for the initial condition

$$
\left[\begin{array}{lll}
\mathbf{U}_{0} & ; & \lambda_{0}
\end{array}\right]=\left[\begin{array}{lllll}
1 & 0 & -1 & ; & 1
\end{array}\right]
$$

From Eq. (16), the desired augmented matrix based on the condition is calculated as

$$
[\widetilde{\mathbf{W}} \quad ; \quad \tilde{\mathbf{F}}]=\left[\begin{array}{ccccc}
1 & 2 & -17 & ; & 94 / 907 \\
1 & 4 & -5 & ; & 3835 / 1457 \\
1 & 0 & -1 & ; & 1
\end{array}\right]
$$

Solving this system, the unknown coefficient matrix is obtained as

$$
\mathbf{A}=\left[\begin{array}{lll}
1202 / 1071 & 568 / 1071 & 450 / 3679
\end{array}\right]^{\mathrm{T}}
$$


СВÜ Fen Bil. Dergi., Cilt 13, Sayı 1 2017, 39-49 s

Therefore, we obtain the approximate solution $y_{2}=1+1.0607 x+0.4893 x^{2}$

For $N=3,4,5$, the obtained approximate solutions are

$$
\begin{aligned}
y_{3} & =1+1.1220 x+0.6114 x^{2}+0.0475 x^{3}, \\
y_{4} & =1+1.0829 x+0.6477 x^{2}+0.1602 x^{3} \\
& +0.0461 x^{4}, \\
y_{5} & =1+0.9996 x+0.5000 x^{2}+0.1690 x^{3} \\
& +0.0420 x^{4}+0.0058 x^{5} .
\end{aligned}
$$

Taking $N=2,3,4,5$, the obtained approximate solutions are compared with the exact solutions in Fig. 5. The absolute errors are demonstrated in Table 3. The graphics of the residual error functions are shown in Fig. 6

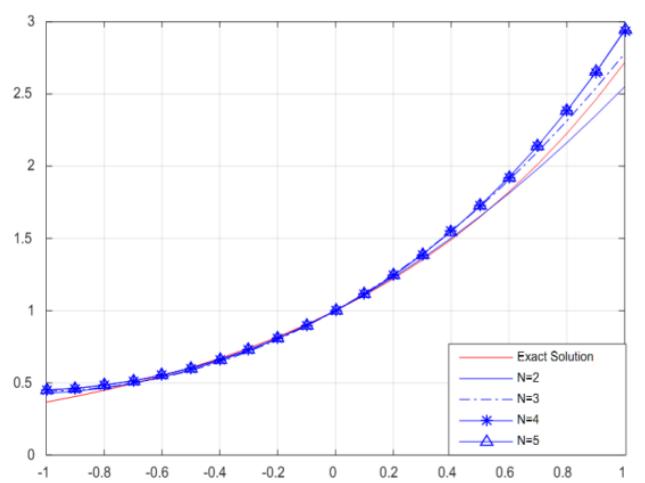

Fig.5. Numerical and exact solutions of Example 3 for $\mathrm{N}=2,3,4,5$.

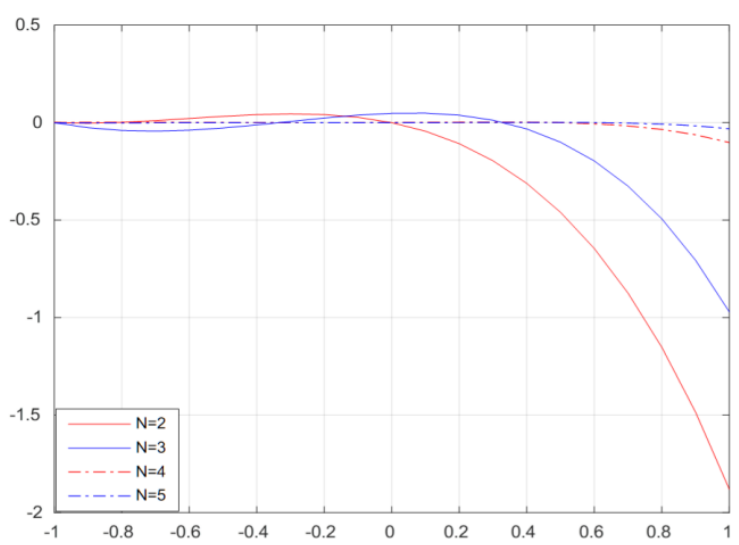

Fig. 6. Residual error functions of Example 3 for $\mathrm{N}=2,3,4,5$.
CBU J. of Sci., Volume 13, Issue 1, 2017 p 39-49

Table 3 . Comparison of the absolute errors of Example 3 for $\mathrm{N}=2,3,4,5$.

\begin{tabular}{cccccc}
\hline$x_{i}$ & $y\left(x_{i}\right)=e^{x_{i}}$ & $\left|e_{2}\left(x_{i}\right)\right|$ & $\left|e_{3}\left(x_{i}\right)\right|$ & $\left|e_{4}\left(x_{i}\right)\right|$ & $\left|e_{5}\left(x_{i}\right)\right|$ \\
\hline-1 & 0.3679 & 0.0607 & 0.0740 & $8.2 \mathrm{e}-02$ & $2.79 \mathrm{e}-04$ \\
-0.5 & 0.6065 & 0.0146 & 0.0206 & $3.2 \mathrm{e}-03$ & $1.19 \mathrm{e}-05$ \\
0 & 1 & 0 & 0 & 0 & 0 \\
0.5 & 1.6487 & 0.0040 & 0.0711 & $7.7 \mathrm{e}-02$ & $9.9 \mathrm{e}-06$ \\
1 & 2.7183 & 0.1683 & 0.0626 & $2.18 \mathrm{e}-02$ & $1.9 \mathrm{e}-03$ \\
\hline
\end{tabular}

\section{Conclusion}

In this study, a numerical method for solving high-order linear differential-difference equations with residual error estimation under mixed conditions is developed. Also, an approximate solution in terms of the Gegenbauer series is obtained by using Gegenbauer polynomials. An error analysis based on residual function is carried out to show the accuracy of the results. It is observed from the tables and figures that the error estimations are very effective. When the exact solution of the problem is not known, the error of the solution can be approximately computed by means of this residual function. In addition, we compared the numerical values of the approximate solutions obtained by the method in tables and figures. These comparisons and the error estimations show that the suggested method is quite effective. 
CBÜ Fen Bil. Dergi., Cilt 13, Say1 1 2017, 39-49 s

\section{References}

[1] Iserles, A. On Neutral FunctionalDifferential Equations with Proportional Delays. J. Math. Anal. Appl. 1997; 207, 73-95.

[2] Wang, W.S.; Li, S. F. On the one-leg Qmethods for solving nonlinear neutral functional differential equations. Appl. Math. Comput. 2007; 193(1), 285-301.

[3] Kuang, Y.; Feldstein, A. Monotonic and Oscillatory solutions of a linear neutral delay equations with infinite lag, SIAM J. Math. Anal. 1990; 21(6), 1633-1641.

[4] Cahlon, B.; Schmidt, D. Stability criteria for certain high even order delay differential equations. J. Math. Anal. Appl. 2007; 334, 859875.

[5] El-Khatib, M.A. Convergenue of the spline function for functional differential equation of neutral type. Intern. J. Comput. Math. 2003; 80(11), 1437-1447.

[6] Rashed, M.T. Numerical solution of functional differential,integral and integrodifferential equations. Appl. Math. Comput. 2004; 156, 485-492.

[7] Cheng, K.; Chen, Z.; Zhang, Q. An approximate solution for a neutral functional differential equation with proportional delays. Appl. Math. Comput. 2015; 260, 27-34.

[8] Bhrawy, A.H.; Assas, L.M.; Tohidi, E.; Alghamdi, M.A. A Legendre-Gauss collocation method for neutral functional differential equations with proportional delays. Advances in Difference Equations. 2013; 2013, 63.

[9] Heydari, M.; Loghmani, G.B.; Hosseini, S.M. Operational matrices of Chebyshev cardinal functions and their aplication for solving delay differential equations arising in elektrodynamics with error estimation. Appl. Math. Model. 2013; 37, 7789-7809.
CBU J. of Sci., Volume 13, Issue 1, 2017 p 39-49 [10] Işik, O.R.; Güney, Z.; Sezer, M. Bernstein series solutions of pantograph equations using polynomial interpolation. Journal of Difference Equations and Applications. 2012; 18(3), 357374.

[11] Gülsu, M.; Öztürk, Y.; Sezer, M. A new Chebyshev polnomial approximation for solving delay differential equations. Journal of Difference Equations and Applications. 2012; 18(6), 1043-1065.

[12] Gürbüz, B.; Sezer, M.; Güler, C. Laguerre collocation method for solving Fredholm integro- differential equations with functional arguments. J. Appl. Math. 2014; (2014) Article ID 682398,12 pages.

[13] Yüzbaşı, Ş.; Gök, E.; Sezer, M. Laguerre matrix method with the residual error estimation for solutions of a class of delay differential equations. Math. Meth. Appl. Sci. 2014; 37(4), 453-463.

[14] Şahin, N.; Yüzbaşı, Ş.; Sezer, M. A Bessel polynomial approach for solving general linear Fredholm integro-differential-difference equations. Int. J. Comput. Math. 2011; 88, 30933111.

[15] Sezer, M.; Gülsu, M. Solving high-order linear differential equations by a Legendre matrix method based on hybrid Legendre and Taylor polynomials. Numerical Methods for Partial Differential Equations. 2010; 26, 647661.

[16] Gülsu, M.; Sezer, M. A Taylor collocation method for solving high-order linear pantograph equations with linear functional argument. Numerical Methods for Partial Differential Equations. 2011; 27, 1628-1638.

[17] Gülsu, M.; Sezer, M. A method for the approximate solution of the high-order linear difference equations in terms of Taylor 
CBÜ Fen Bil. Dergi., Cilt 13, Sayı 1 2017, 39-49 s

polynomials. International Journal of Computer Mathematics. 2004; 82(5), 629-642.

[18] Kim, D.S.; Kim, T.; Rim, S.H. Some identities involving Gegenbauer polynomials. Advance in Difference Equations. 2012; 2012: 219.

[19] Khan, S.; Al-Gonah, A.A.; Yasmin, G. Generalized and mixed type Gegenbauer polynomials. J. Math. Anal. Appl. 2012; 390, 197-207.

[20] Lewanowicz, S. Results on the associated Jacobi and Gegenbauer polynomials. Journal of Computational and Applied Mathematics. 1993; 49, 137-143.

[21] Kürkçü, Ö.K.; Aslan, E.; Sezer, M. A numerical approach with error estimation to solve general integro-differential-difference equations using Dickson polynomials. Applied Mathematics and Computation. 2016; 276, 324339. 